\title{
Kindler, Lars (2018): Zur Steuerungskraft der Raumordnungsplanung. Am Beispiel akzeptanzrelevanter Konflikte der Windenergieplanung
}

\section{Baden-Baden: Nomos, 418 Seiten. = Leipziger Schriften zum Umwelt- und Planungsrecht 34.}

https://doi.org/10.2478/rara-2019-0006

Eingegangen: 10. Oktober 2019; Angenommen: 29. Januar 2019

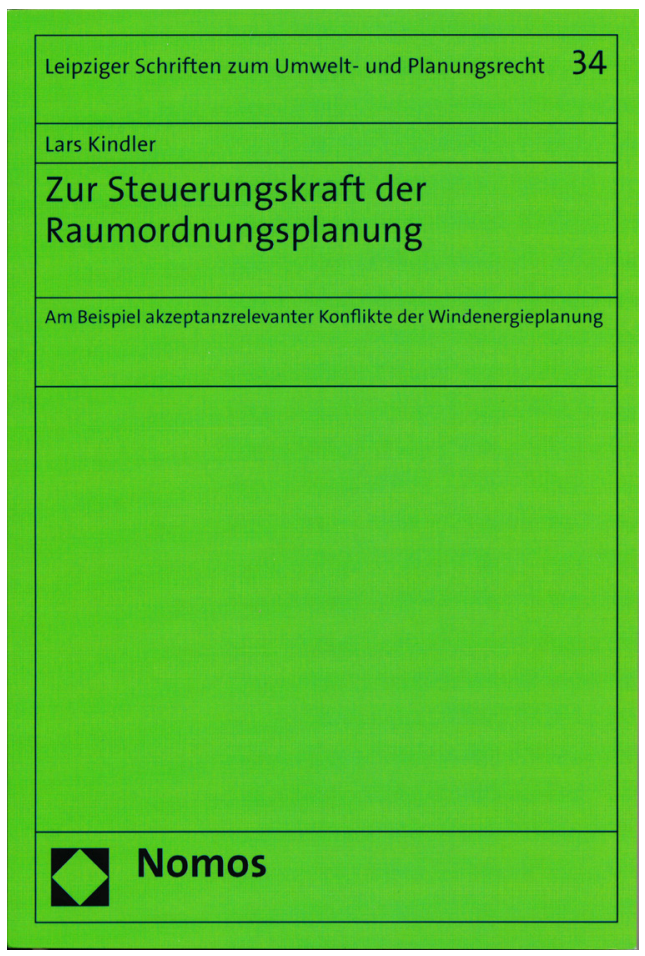

Nicht nur die viel beschworenen Vorgänge um die Realisierung des (Bahn-)Projekts „Stuttgart 21“ und ähnlicher Vorhaben, sondern auch Konflikte im leitungsgebunde- nen Bereich, aktuell etwa bei den Trassen- und Leitungsfestlegungen für den Transport offshore gewonnenen Stroms in den Süden der Republik, haben die Erkenntnis nach sich gezogen, dass neben allen rechtlichen und gerichtlichen Kautelen bzw. Entscheidungen die Akzeptanz in der Bevölkerung zentrale Bedeutung für die Verwirklichungschancen infrastruktureller Maßnahmen hat. Indem fachgesetzlich geplante oder gesteuerte Projekte bei den Bürgern regelmäßig in den Verdacht einseitiger, nämlich trägerorientierter Ausrichtung geraten, liegt es nahe, die notwendige Akzeptanzgewinnung durch Einsatz der nicht fachlich, sondern neutral und auf Konfliktmittlung ausgerichteten Raumordnung zu erreichen. Da es hierzu bislang kaum rechtliche Untersuchungen gibt, erscheint es verdienstvoll, dass sich die Dissertation von Lars Kindler, die bei Wolfgang Köck, Leiter des Departments Umwelt- und Planungsrecht am HelmholtzZentrum für Umweltforschung (UFZ) in Leipzig, entstanden ist, eben diesem Thema widmet, und zwar anhand der Windenergieplanung und dabei beschränkt auf materiell-rechtliche Fragestellungen sowie - instrumentell - auf die Raumordnungsplanung.

Dabei sind als Maßstab der Überprüfung zunächst Begriff und Verständnis der Akzeptanz zu klären sowie die spezifisch akzeptanzrelevanten Konflikte in der bei-

\footnotetext{
*Corresponding author: Prof. Dr. Wilfried Erbguth, Friedrich-Franz-Straße 38, 18119 Rostock-Warnemünde,

E-mail: wilfried.erbguth@uni-rostock.de
} 
spielgebenden Windenergienutzung zu beleuchten. Das geht die Arbeit denn auch in ihrem ersten Schritt an. Sodann wird unter Auswertung von Rechtsprechung und Literatur das nicht unkomplizierte Rechtsregime für die diesbezügliche planerische Standortsteuerung durch Raumordnungsplanung dargestellt. Das umfangreichste, weil dem thematischen Kern der Untersuchung gewidmete dritte Kapitel wendet sich sodann der Steuerungskraft des planerischen Instrumentariums der Raumordnung zu. Hier geht es unter anderem um Vorgaben kompetenzieller Art, um das grenzziehende kommunale Selbstverwaltungsrecht - und sodann in konkretisierender Ableitung um die Konsequenzen für bestimmte Festsetzungen, denen zentrale Akzeptanzrelevanz zugeschrieben wird (Höhenbeschränkungen, Bedingungen und Befristungen bezüglich Repowering (Erneuerung alter Windkraftanlagen) und finanziell bürgergerichtete Beteiligungsmodelle).

Hervorgehoben sei Folgendes: Im Zusammenhang mit dem Akzeptanzverständnis erscheint die aus der Soziologie übernommene Unterscheidung zwischen gesellschaftlicher und lokaler Akzeptanz als aussagekräftiges Differenzierungsmodell für die nachfolgenden, gerade auch rechtlichen Detailüberprüfungen sinnvoll. Hinterfragen lässt sich hingegen die Annahme, dass akzeptanzmindernde oder -ausschließende Konflikte nicht durch ein besseres Verfahrensmanagement, sondern nur materiell-rechtlich bewältigt werden können - ist doch das Verfahrensstadium durch die prinzipiell noch offene Entscheidungsfindung gekennzeichnet. Verfolgenswert erscheint im Weiteren, die Details der Standortbestimmung von Vorhaben akzeptanzorientiert festzulegen. Im - nachfolgenden - Zusammenhang rechtlicher Vorgaben für die Standortsteuerung bei Windenergieanlagen durch Raumordnungsplanung wird zu Recht die zentrale Rolle des $\S 35$ Abs. 3 S. 3 BauGB hervorgehoben und auch, dass die hierauf gerichtete Rechtsprechung, insbesondere des Bundesverwaltungsgerichts, erhebliche Verunsicherungen in der Praxis bei der Umsetzung des Planungsvorbehalts nach sich gezogen hat. Dieses Richterrecht findet sich im Näheren mit guten Gründen kritisiert. Dass die Raumordnung über jene Vorschrift auf ein stärkeres Maß an Vorhabenbezug verpflichtet wird, wird gesehen, hätte aber - gerade unter Aspekten der Akzeptanz - stärker problematisiert werden können. Im Kapitel zur Steuerungskraft der Raumordnung(splanung) findet sich als Konsequenz des verfassungsrechtlich gewährleisteten kommunalen Selbstverwaltungs- und (damit) Planungsrechts die Einräumung hinreichender gemeindlicher Konkretisierungs- und Ausgestaltungsmöglichkeiten gegenüber der Windenergieplanung durch Raumordnung angemahnt. Ob freilich $\S 35 \mathrm{Abs}$. $3 \mathrm{~S}$. 3 BauGB eine qualifizierte Planungsermächtigung für die bodenrechtliche Wirkung raumordnerischer Festlegungen darstellt, lässt sich angesichts verfassungsrechtlicher Grenzsicherungen und Aufgabenverteilungen durchaus hinterfragen. Ähnliches gilt - im konkreteren Zusammenhang der (planerischen) Festlegungen mit Akzeptanzrelevanz - gegenüber der Annahme, eine qualifizierte Planungsermächtigung, möglichst bodenrechtlicher Art, reiche aus, um raumordnungsplanerische Höhenbeschränkungen für Windenergieanlagen kompetenzgerecht erscheinen zu lassen. Repoweringfestlegungen werden aus allgemeinen rechtlichen Gründen (Wettbewerb, Eigentumssituation) als nicht unbedenklich eingestuft. Was die finanzielle Beteiligung von (Gemeinden und) Bürgern an Windkraftprojekten anbelangt, so hätten die Zweifel an der Zulässigkeit hierauf gerichteter raumplanerischer Steuerung (noch) deutlicher ausfallen können. Entsprechendes lässt sich gegenüber dem abschließend als Fortentwicklung betonten stärkeren Vorhabenbezug der Raumordnung anmerken, und zwar in seinem durchaus gesehenen Spannungsfeld zur Raumordnung als „Planung der Planungen“.

Insgesamt zeichnet sich die Untersuchung durch gute Lesbarkeit und eine breit angelegte Verarbeitung von Rechtsprechung und Schrifttum aus, Letzteres auch über den rechtswissenschaftlichen Bereich hinaus. Argumentativ hätte man sich zuweilen eine deutlichere Trennung zwischen juristischen Gründen und solchen des außerjuristischen Bereichs gewünscht. Auch wäre konzeptionell daran zu denken gewesen, die Akzeptanzsicherung durch Raumordnung stärker auf deren koordinierenden und damit verobjektivierenden Aufgabengehalt zu fokussieren. Insgesamt vermittelt die Abhandlung aber interessante Ansätze für die Bewältigung einer zunehmend problematischen Situation im Verhältnis Staat-Bürger. 